\title{
Acute ST segment elevation MI with Normal Coronaries
}

\author{
Barakoti M, Jha SC, Acharya SM, Paudel CM
}

\section{Address for Correspondence:}

Manmohan Cardiovascular Thoracic and Transplant Centre (MCVTC)

Tribhuvan University, Institute of Medicine, Maharajgunj, Kathmandu, Nepal

drmuraribarakoti@gmail.com

\section{Abstract}

A 45 year-old man with no history of cardiac disease presented to the emergency department with typical angina chest pain of $>24$ hours duration. He was not thormbolyzed due to late presentation. Having elevated troponin and CK-MB levels and an electrocardiogram demonstrating anterolateral ST segment elevation and persistent of angina pain prompted coronary angiography which could be done only after 3 weeks of symptom onset due to financial constraint; which revealed coronary vessels free of significant disease. An echocardiogram showed dilatation of all cardiac chambers with hypokinesia of anterior wall and mid-septum and akinesia of apex. There was moderate mitral regurgitation and moderate tricuspid regurgitation and moderate systolic dysfunction.

A number of conditions can lead to ST segment elevation MI with normal epicardial coronary anatomy. We report a case possibly due to autothrombolysis. Treatment to such cases would be symptomatic drug therapy with reassurance and risk factor reduction. 


\section{Case}

A 45 years old man, a bus driver of a public vehicle, was seen in the emergency department of Manmohan Cardiovascular Thoracic and Transplant Centre (MCVTC), Maharajgunj, Kathmandu with the complaint of substernal chest pain.

The patient had been well until one day before the presentation, while he was staying at home in at around 10:00 am, pain in the substernal area developed suddenly. The pain was radiating to the both of his limbs. He vomited 4 times and felt nauseated. Since the same duration he felt shortness of breath on minimal exertion like walking to the bathroom.

He felt that the symptoms are due to minor illness and remained at home. He continued to feel uneasiness that day. He had orthopnea and needed extra pillows to get rid of dyspnea. He presented next day to ER, where he was evaluated. On examination, he was tachycardic with pulse rate of 100 beats per minute and his Blood Pressure was $100 / 80 \mathrm{~mm}$ of $\mathrm{Hg}$ right arm in supine position. He had S3 gallop and bibasal cracles on ausculatation. Rests of the clinical examination were normal.

An electrocardiogram (ECG) showed a normal sinus rhythm, with rate of 136 beats per minute, and ST-segment elevation of up to $0.3 \mathrm{mV}$ in leads $\mathrm{V} 1$ through $\mathrm{V} 6, \mathrm{I}$, and $\mathrm{aVL}$, and QS pattern in V1 through V6. Oxygen, acetylsalicylic acid, clopidrogel, intravenous frusemide were administered. Enalapril and subcutaneous enoxaparine were also given. Patient was not thrombolysed due to late presentation (>24 hours). Patient was getting much relief after treatment and got admitted in CCU.

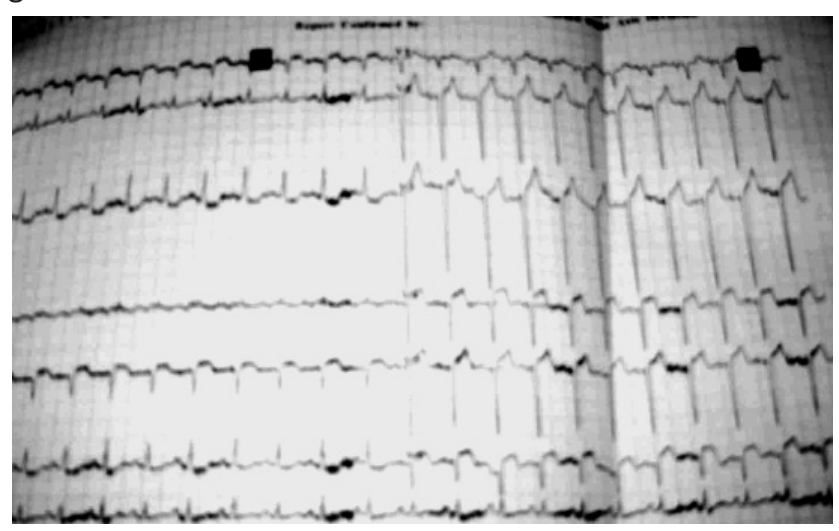

Figure 1. ECG at time of presentation to ER

He was not hypertensive and not a diabetic. He had no previous symptoms suggesting coronary artery disease, peripheral vascular artery or cerebrovascular disease. He was taking no medications and had no allergies. He was teetotaler and non smoker. The family history was non contributory.
The testing for Troponin I was positive by rapid antigen test, CK-MB level was $330 \mathrm{U} / \mathrm{l}$, Hemoglobin was $18.1 \mathrm{gm} / \mathrm{dL}$, total white cell count $19,500 / \mathrm{cmm}$ with $90 \%$ neutrophils. Serum electrolytes, plasma glucose level and urine routine examination were normal.

CK-MB reduced to $235 \mathrm{U} / \mathrm{L}$ on $2^{\text {nd }}$ day of onset of symptoms and reduced to $54 \mathrm{U} / \mathrm{L}$ on $3^{\text {rd }}$ day. Lipid profile showed total cholesterol of $3.7 \mathrm{mmol} / \mathrm{L}$, LDL-cholesterol $2.2 \mathrm{mmol} / \mathrm{L}$, $\mathrm{HDL}$ - cholesterol $1.1 \mathrm{mmol} / \mathrm{L}$ and triglyceride $0.7 \mathrm{mmol} / \mathrm{L}$. Serum urea and electrolytes remained normal throughout the hospital stay. White cell count normalized on $10^{\text {th }}$ day.

During the stay he developed hospital acquired pneumonia and recurrent angina. The patient got better with intravenous Cefepime and Amikacin and as required Glyceryl Trinitrate sublingual spray. Fundoscopy revealed normal examination.

Echocardiography showed dilatation of all cardiac chambers with hypokinesia of anterior wall and midseptum and akinesia of apex. There was moderate mitral regurgitation and moderate tricuspid regurgitation. Right ventricular systolic pressure was calculated to be $65 \mathrm{~mm}$ $\mathrm{Hg}$. Left ventricular ejection fraction was $44 \%$. There was no mass, thrombus or pericardial effusion.

Subsequent ECG showed gradual resolution of ST-segment, and QS pattern was persisting in V1 through V6. Later ECG showed T-wave inversion in lateral leads.

Patient was managed conservatively and was improving slowly and gradually. He was gradually mobilized and discharged after $23^{\text {rd }}$ days hospital stay. He was advised to take Acetylsalicylic Acid, clopidrogrel, atorvastatin, metoprolol succinate, enalapril and nitrate. He was referred to Shahid Gangalal National Heart Centre for coronary angiography. The coronary angiography was done on $3^{\text {rd }}$ week of onset of symptoms. The report showed completely normal epicardial coronary arteries.

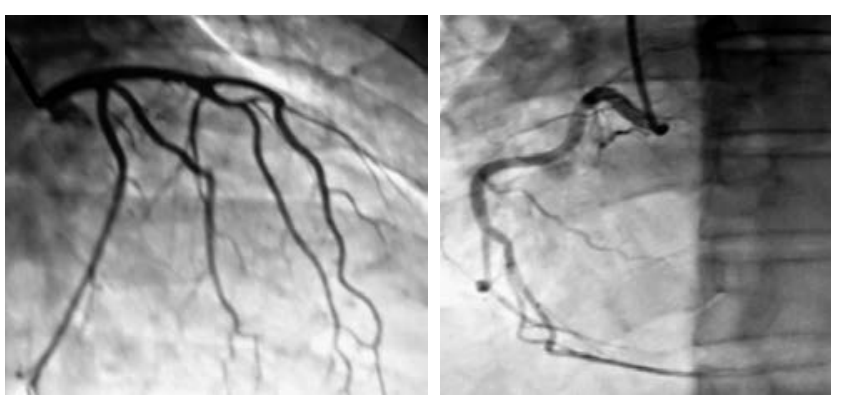

Figure 2. Angiogram report

\section{Discussion}

What could be the possibility of normal coronary arteries?

Approximately $4-7 \%$ of all patients with acute myocardial infarction and nearly four times this percentage of patients younger than age 35 years do not have atherosclerotic 
coronary artery disease as demonstrated by coronary angiography or at necropsy or both [1-3]. Coronary angiography simply represents an image of the lumen, so the specificity for etiology of the coronary artery disease is extremely low. Review of necropsy studies shows that approximately $95 \%$ of patients with fatal acute myocardial infarction have at least one major epicardial coronary artery with severe luminal narrowing or total occlusion. The remaining $5 \%$ of these patients have normal major epicardial coronary artery.

Of the $95 \%$ of the patients with severe coronary artery luminal narrowing, 95\% have typical atherosclerotic plaque with a superimposed thrombus in $85 \%$. The remaining $5 \%$ of patients with severe coronary luminal narrowing have a host of etiology, including coronary arteritis, trauma, systemic metabolic disorders (mucopolysaccharidosis, amyloidosis, Fabry's disesase, homocystinuria), intimal fibrous proliferation, and coronary emboli.

Of the $5 \%$ of patients seen at necropsy after acute myocardial infarction with normal epicardial coronary arteries, 50- 60\% likely represent clinical coronary spasm, but the remaining $40-50 \%$ represent a combination of congenital coronary artery anomalies, spontaneous recanalization, and mismatches of coronary supply and myocardial demand.

There are relatively few necropsy reports of patients with acute myocardial infarction who had angiographically normal coronary arteries and normal coronary arteries at necropsy $[4,5]$. But these do occur. The possible explanations for these include coronary artery spasm, coronary artery disease in vessels too much small to be visualized angiographically and coronary artery thrombus or emboli with subsequent clot lysis.

The possibility in our presented case could be coronary artery thrombosis and spontaneous clot lysis and recanalization a condition termed as cardiac syndrome $\mathrm{X}$. Though, we have not excluded coronary artery spasm by inducing with ergonovine provocation test [6]. The chest pain is usually indistinguishable with traditional angina caused by obstructive coronary disease and is, therefore, considered a diagnosis of exclusion. When intravascular ultrasonography (IVUS) studies have been performed in these patients a spectrum of finding ranging from normal vessels to intimal thicking to nonobstructive atheromatous plaque has been reported [7].

The Possible mechanisms for the absence of significant coronary disease include rapid clot lysis, coronary vasospasm (variant angina), cocaine use, inherited thrombophilia, and coronary microvascular disease. In favor of the last mechanism is the observation in a report from the TIMI IIIA trial that approximately one-third of these patients had abnormally slow angiographic filling (TIMI flow grade 2 or less) [8].

The exact basis for the signs and symptoms of this condition has not been elucidated. Some general statements can be made, however, regarding the clinical profile of these patients. Syndrome $X$ has an increased occurrence in women (3:1 preponderance), both pre- and post-menopausal [9]. Endothelial dysfunction, microvascular ischemia, and abnormal pain perception have all been implicated for the etiology of the disorder. Endothelial dysfunction as demonstrated by abnormal coronary flow reserve (CFR), single photon emission computed tomography stress and positron emission tomography stress testing is common in these patients. In addition, behavioral and psychiatric conditions often exist.

Another possibility could be Takotsubo syndrome, also known as LV ballooning syndrome or stress induced cardiomyopathy. It presents with sudden onset chest pain, ECG changes with ST elevation, mimicking acute myocardial infarction, usually in the setting of severe emotional distress and catecholamine surge. The angiogram shows normal coronary artery and diagnosis is made on the typical appearance by LV ventriculogram or echocardiogram with basal hyperkinesis and severe apical systolic wall motion abnormality [10]. Most patients recover LV function and require only hemodynamic and pharmacological support.

Thus sometimes we consider the coronary artery disease to be the causative factor for acute myocardial infarction and coronary angiography comes to be completely normal as our case. In such a situation, the treatment consists of symptomatic treatment with drugs, reassurance and aggressive reduction of risk factors. In developing countries like Nepal for logistic and economic reason invasive therapy is still a challenge, yet when done may come normal. This may bring tremendous stress to the patient and bewildering to the physician. More investigation and development of strategy to diagnose and treat these disorders in the future will be helpful. 


\section{References:}

1. Waller BF. Atherosclerotic and non-atherosclerotic coronary artery factors in acute myocardial infarction. In: Pepine CJ, ed. Acute Myocardial Infarction. Philadelphia, PA:FA Devis; 1989:29- 104.

2. Cheitlin MD, McAllister HA, deCastro CM. Myocardial infarction without atherosclerosis. JAMA. 1975; 231:951- 959.

3. Eliot RS, Baroldi G. Necropsy studies in myocardial infarction with minimal or no coronary luminal reduction due to atherosclerosis. Circulation. 1974;49:1127- 1131.

4. Virmani R, Forman MB, eds. Nonatherosclerotic Ischemic Heart Disease. New York, NY: Raven Press; 1989:1-428.

5. Friedberg $\mathrm{CK}$, Horn $\mathrm{H}$. Acute myocardial infarction not due to coronary artery occlusion. JAMA. 1939; 112:1675- 1679.

6. Bugairdini R, Bairey Merz CN. Angina with 'normal' coronary artery: A changing philosophy. JAMA .2005;290:477.

7. ErbelR, GeJ. Bockisch A, et al. value of intracoronary ultrasound and Doppler in the differentiation of angiographycially normal coronary arteries: a prospective study in patients with angina pectoris. Eur Heart Journal. 1996;17:880- 889.

8. Diver DJ, Bier JD, Ferreira PE, et al. Clinical and arteriographic characterization of patients with unstable angina without critical coronary arterial narrowing (from the TIMI-IIIA Trial). Am J Cardiol. 1994 ;74(6):531-7.

9. Kaski JC, Rosano GMC, Collins P, et al. Cardiac syndrome X: clinical characteristics and left ventricular function- long term follow up study. J Am Coll Cardiol. 1995;25:807- 814.

10. Bybee KA, Kara T, Prasad A, et al. Systematic review: transient left ventricular apical ballooning: a syndrome that mimics STsegment elevation myocardial infarction. Ann Intern Med. 2004;141:858- 865 . 